\title{
Deconfinement Phase Transition in an Expanding Quark system in Relaxation Time Approximation
}

\author{
Zhenwei Yang and Pengfei Zhuang \\ Physics Department, Tsinghua University, Beijing 100084, China
}

We investigated the effects of nonequilibrium and collision terms on the deconfinement phase transition of an expanding quark system in Friedberg-Lee model in relaxation time approximation. By calculating the effective quark potential, the critical temperature of the phase transition is dominated by the mean field, while the collisions among quarks and mesons change the time structure of the phase transition significantly. PACS : 05.60. + w, 52.60. + h, 12.38.Mh

\section{INTRODUCTION}

It is generally accepted that the most interesting quantum chromodynamics (QCD) phase transition in hot and dense nuclear matter is the deconfinement phase transition between normal nuclear matter and quark-gluon plasma (QGP), where quarks and gluons are no longer confined. The theoretical and experimental investigation of QGP is one of the most challenging problems in high energy physics. It is widely believed that QGP phase can be formed in ultra-relativistic heavy-ion collisions.

Due to the difficulty of non-perturbative treatment in QCD, various models have been considered in the study of the phase transition among which Friedberg-Lee model [1], also referred as nontopological soliton model, has been widely discussed in the past two decades, see for instance $[2,3]$ and refs. therein. In this model, the non-perturbative dynamics responsible for confinement in QCD is simulated in terms of a non-linear coupling to a scalar field $\sigma$. It shows an intuitive mechanism for the deconfinement phase transition. In vacuum state, the physical value of $\sigma$ is large and the quark mass is more than $1 \mathrm{GeV}$, so that the effective heavy quarks have to be confined in hadron bags [1]. With increasing temperature and/or density of the system, the physical value of $\sigma$ and in turn the effective quark mass drops down, the thermodynamic motion leads to a deconfinement of the effective light quarks.

Wilets and his cooperators [3,4] did a great deal of work on the properties of Friedberg-Lee model, mostly in mean field approximation and in vacuum state. It is proved very successful in describing the static properties of the nucleon. During the past years, Friedberg-Lee model was extended to finite temperature and density to study deconfinement phase transition [5-8]. Similar to most of these investigations in the frame of finite tem- perature field theory, the temperature and density effect on the phase transition is based on the assumption of a thermalized plasma phase. While one can use various parameters and take different treatments, the critical temperature of the deconfinement phase transition in the model is limited in the region of 80 to $120 \mathrm{MeV}$ at zero chemical potential, much lower than the prediction in Lattice QCD [9].

Because of the estimated very short lifetime of the heavy ion collision zone, the highly excited particle system may spend a considerable fraction of its life in a nonthermalized, preequilibrium state. The dynamical tool to treat dissipative processes in heavy ion collisions and the approach to local thermal equilibrium is in principle nonequilibrium quantum transport theory. A relativistic and gauge covariant kinetic theory for quarks and partons has been derived [10], both in a classical framework $[11,12]$ and as a quantum kinetic theory [13,14] based on Winger operators defined in eight-dimensional phase space [15]. To solve the quantum kinetic equations as initial problems, the transport and off-shell constraint hierarchies have been established [16-19] in the frame of equal-time Wigner operators. The properties of nucleonnucleon collisions have been explored $[20,21]$ in transport approach of Friedberg-Lee model.

In the present paper, we consider an expanding nonequilibrium system with collision terms in the framework of Friedberg-Lee model to investigate the approach of strong interaction matter toward thermal equilibrium and the deconfinement phase transition during this process. The collision terms will be introduced through a relaxation time approximation in transport equations of quarks and sigmas, and the expansion of the system is simply described by the Bjorken scaling hydrodynamics [22]. We specially focus on the effect of collision terms and non-equilibrium on the deconfinement phase transition, by investigating at what proper time the phase transition occurs, how long it lasts for a first-order transition, and their dependence on the relaxation time.

The outline of the paper is as follows. The full transport equations for quarks and sigmas in relaxation time approximation, and the simplified equations in quasiparticle limit and boost invariance approximation are presented in Section 2. In section 3 we exhibit the numerical results and discussions. Finally a brief summary is given in the last section. 


\section{TRANSPORT EQUATIONS}

The Friedberg-Lee model is defined as [1-3]

$$
\begin{aligned}
\mathcal{L}_{F L} & =\hat{\bar{\psi}}\left(i \gamma^{\mu} \partial_{\mu}-\left(m_{0}+g \hat{\sigma}\right)\right) \hat{\psi}+\frac{1}{2} \partial^{\mu} \hat{\sigma} \partial_{\mu} \hat{\sigma}-U(\hat{\sigma}) \\
U(\hat{\sigma}) & =\frac{a}{2} \hat{\sigma}^{2}+\frac{b}{3 !} \hat{\sigma}^{3}+\frac{c}{4 !} \hat{\sigma}^{4}+B
\end{aligned}
$$

where $\hat{\psi}, \hat{\bar{\psi}}$ and $\hat{\sigma}$ are quark, antiquark and scalar fields, respectively, $m_{0}$ is the current quark mass and chosen to be 0 in the following to simplify the calculations. There are five parameters in the Friedberg-Lee model. $a$ with dimension $L^{-2}, b$ with dimension $L^{-1}$, dimensionless $c$, the coupling constant $g$ between quark and scalar fields, and the bag constant $B$ used to provide a quark confinement potential in mean field approximation in the vacuum. Of the five parameters, two are adjusted to fit the proton size and the nucleon mass, and the others are left to survey, fit and predict physical data. Since the model is an effective one, the values of the parameters depend on the level of approximation employed [23]. Different parameter sets can be found in the book by Wilets [3].

The relativistically covariant quark Wigner operator $\hat{W}_{q}$ and sigma Wigner operator $\hat{W}_{\sigma}$ are the Fourier transform of the corresponding density matrices [15]

$$
\begin{aligned}
\hat{W}_{q}(x, p) & =\int d^{4} y e^{i p y} \hat{\Phi}_{q}(x, y) \\
& =\int d^{4} y e^{i p y} \hat{\psi}\left(x+\frac{y}{2}\right) \hat{\bar{\psi}}\left(x-\frac{y}{2}\right), \\
\hat{W}_{\sigma}(x, p) & =\int d^{4} y e^{i p y} \hat{\Phi}_{\sigma}(x, y) \\
& =\int d^{4} y e^{i p y} \hat{\sigma}\left(x+\frac{y}{2}\right) \hat{\sigma}\left(x-\frac{y}{2}\right) .
\end{aligned}
$$

Calculating the first-order derivatives of $\hat{\Phi}_{q}$ and second-order derivatives of $\hat{\Phi}_{\sigma}$ with respect to $x$ and $y$, and making use of the equations of motion

$$
\begin{aligned}
& \left(i \gamma^{\mu} \partial_{\mu}-\left(m_{0}+g \hat{\sigma}\right)\right) \hat{\psi}=0, \\
& \partial^{\mu} \partial_{\mu} \hat{\sigma}+\frac{\partial U(\hat{\sigma})}{\partial \hat{\sigma}}+g \hat{\bar{\psi}} \hat{\psi}=0
\end{aligned}
$$

for the fields, one obtains evolution equations for the density matrices. After Wigner transform one derives the kinetic equations (for details, see the similar work in [17] for QED)

$$
\begin{aligned}
\left(K_{\mu} K^{\mu}-\Sigma_{\sigma}\right) W_{\sigma}(x, p) & =C_{\sigma}, \\
\left(\gamma^{\mu} K_{\mu}-\Sigma_{q}\right) W_{q}(x, p) & =C_{q}
\end{aligned}
$$

for the Wigner functions $W_{q}(x, p)$ and $W_{\sigma}(x, p)$ which are, respectively, the ensemble average of the Wigner operators,

$$
\begin{aligned}
W_{q}(x, p) & =\int d^{4} y e^{i p y}\left\langle\hat{\psi}\left(x+\frac{y}{2}\right) \hat{\bar{\psi}}\left(x-\frac{y}{2}\right)\right\rangle, \\
W_{\sigma}(x, p) & =\int d^{4} y e^{i p y}\left\langle\hat{\sigma}\left(x+\frac{y}{2}\right) \hat{\sigma}\left(x-\frac{y}{2}\right)\right\rangle .
\end{aligned}
$$

The operators $K_{\mu}, \Sigma_{\sigma}$ and $\Sigma_{q}$ are defined as

$$
\begin{aligned}
& K_{\mu}=p_{\mu}+\frac{i \hbar}{2} \partial_{\mu}, \\
& \Sigma_{\sigma}=\left(a+b \sigma+\frac{c}{2}\left(\left\langle\hat{\sigma}^{\prime} \hat{\sigma}^{\prime}\right\rangle+\sigma^{2}\right)\right) e^{-\frac{i}{2} \hbar \partial_{x}^{\mu} \partial_{\mu}^{p}}, \\
& \Sigma_{q}=\left(m_{0}+g \sigma \cos \left(\frac{\hbar}{2} \partial_{x}^{\mu} \partial_{\mu}^{p}\right)\right)-i g \sigma \sin \left(\frac{\hbar}{2} \partial_{x}^{\mu} \partial_{\mu}^{p}\right),
\end{aligned}
$$

where $\sigma$ and $\hat{\sigma}^{\prime}$ are, respectively, the mean field and quantum fluctuation of the scalar field, $\sigma=\langle\hat{\sigma}\rangle$ and $\hat{\sigma}=\sigma+\hat{\sigma}^{\prime}$. The sigma and quark scalar densities $\left\langle\hat{\sigma}^{\prime} \hat{\sigma}^{\prime}\right\rangle$ and $\langle\hat{\bar{\psi}} \hat{\psi}\rangle$ can be calculated through the Wigner functions

$$
\begin{aligned}
& <\hat{\sigma}^{\prime} \hat{\sigma}^{\prime}>=\int \frac{d^{4} p}{(2 \pi)^{4}} W_{\sigma}(x, p), \\
& <\hat{\bar{\psi}} \hat{\psi}>=\operatorname{Tr} \int \frac{d^{4} p}{(2 \pi)^{4}} W_{q}(x, p) .
\end{aligned}
$$

In relaxation time approximation the collision terms $C_{\sigma}$ and $C_{q}$ can be written as

$$
\begin{aligned}
& C_{\sigma}=-i \hbar p^{\mu} u_{\mu} \frac{W_{\sigma}-W_{\sigma}{ }^{t h}}{\theta}, \\
& C_{q}=-\frac{i \hbar}{2} \gamma^{\mu} u_{\mu} \frac{W_{q}-W_{q}^{t h}}{\theta},
\end{aligned}
$$

where $u_{\mu}$ is the four-velocity of the hot medium formed by quarks and sigmas, and $\theta$ is the relaxation time. It is necessary to note that the space-time derivative $\partial_{x}$ in the self-energies $\Sigma_{\sigma}$ and $\Sigma_{q}$ works only the mean field $\sigma$ and scalar density $\left\langle\hat{\sigma}^{\prime} \hat{\sigma}^{\prime}\right\rangle$ on its left.

Making ensemble average of the Klein-Gordon equation in (3), one gets the equation of motion for the condensate $\sigma$,

$$
\partial^{\mu} \partial_{\mu} \sigma+\frac{\partial U_{e f f}}{\partial \sigma}=0
$$

with the definition of the effective confinement potential $U_{\text {eff }}\left(\sigma,\left\langle\hat{\sigma}^{\prime} \hat{\sigma}^{\prime}\right\rangle,\langle\hat{\bar{\psi}} \hat{\psi}\rangle\right)$ at finite temperature

$$
\frac{\partial U_{e f f}}{\partial \sigma}=\frac{\partial U(\sigma)}{\partial \sigma}+\frac{\left\langle\hat{\sigma}^{\prime} \hat{\sigma}^{\prime}\right\rangle}{2}(b+c \sigma)+g\langle\hat{\bar{\psi}} \hat{\psi}\rangle
$$

which depends on not only the mean field but also the sigma and quark scalar densities. The Eqs.(4) and (9) together determine the sigma and quark scalar distributions and the condensate $\sigma$ self-consistently.

From the first-order derivative of the effective potential $U_{\text {eff }}$, Eq.(10), it can be defined as 


$$
\begin{aligned}
& U_{e f f}\left(\sigma,\left\langle\hat{\sigma}^{\prime} \hat{\sigma}^{\prime}\right\rangle,\langle\hat{\bar{\psi}} \hat{\psi}\rangle\right)= \\
& U(\sigma)+U_{\sigma}\left(\sigma,\left\langle\hat{\sigma}^{\prime} \hat{\sigma}^{\prime}\right\rangle\right)+U_{q}(\sigma,\langle\hat{\bar{\psi}} \hat{\psi}\rangle), \\
& U_{\sigma}\left(\sigma,\left\langle\hat{\sigma}^{\prime} \hat{\sigma}^{\prime}\right\rangle\right)=\int_{0}^{\sigma} d \tilde{\sigma} \frac{1}{2}\left\langle\hat{\sigma}^{\prime} \hat{\sigma}^{\prime}\right\rangle(\tilde{\sigma}, x)(b+c \tilde{\sigma}), \\
& U_{q}(\sigma,\langle\hat{\bar{\psi}} \hat{\psi}\rangle)=\int_{0}^{\sigma} d \tilde{\sigma} g\langle\hat{\bar{\psi}} \hat{\psi}\rangle(\tilde{\sigma}, x) .
\end{aligned}
$$

The two extra terms $U_{\sigma}$ and $U_{q}$ arise from the collective motion of the quarks and sigmas. In the vacuum without collective motion the confinement potential $U_{\text {eff }}=U$ has two minima, $\sigma_{p e r}=0$ corresponding to the perturbative vacuum and $\sigma_{p h y} \neq 0$ to the physical vacuum, see the dashed line in Fig.1. From the definition of the bag constant, the energy density difference between the perturbative and physical vacua, $B$ is determined by $U\left(\sigma_{p h y}\right)=0$,

$$
-B=\frac{a}{2 !} \sigma_{p h y}^{2}+\frac{b}{3 !} \sigma_{p h y}^{3}+\frac{c}{4 !} \sigma_{p h y}^{4} .
$$

The bag constant $B$ depends strongly on the parameters $a, b, c$ and $g$. For various reasonable sets of parameters, however, $B$ is around $20 \mathrm{MeV} / \mathrm{fm}^{3}$ [3]. As discussed in [5], it is the small bag constant $B$ which leads to the low critical temperature of deconfinement phase transition in Friedberg-Lee model.

An important aspect of the covariant kinetic theory is that the complex kinetic equation can be split up into a constraint and a transport equation $[13,14,17-19]$, where the former is a quantum extension of the classical on-shell condition, and the latter is a covariant generalization of the Vlasov-Boltzmann equation. The complementarity of these two ingredients is essential for a physical understanding of quantum kinetic theory. The quark Wigner function itself has no direct physical analogue since it is not a self-hermitian function. After the Lorentz decomposition [13,14,17-19], the kinetic equation for quark is changed into 16 transport equations and 16 constraint equations for the 16 Lorentz components of the quark Wigner function. In classical limit with $\hbar=0$, the constraint equations for quark and sigma are reduced to

$$
\begin{gathered}
\left(p^{2}-m_{\sigma}^{2}\right) W_{\sigma}(x, p)=0, \\
\left(p^{2}-m_{q}^{2}\right) W_{q}(x, p)=0
\end{gathered}
$$

with effective sigma mass and quark mass

$$
\begin{aligned}
& m_{\sigma}^{2}=a+b \sigma+\frac{c}{2}\left(\left\langle\hat{\sigma}^{\prime} \hat{\sigma}^{\prime}\right\rangle+\sigma^{2}\right), \\
& m_{q}=m_{0}+g \sigma,
\end{aligned}
$$

and the 16 spinor components are no longer fully independent. Only the quark number density $f_{q}$ and the spin density $\vec{g}_{0}$ are the fundamental elements and the other components can be expressed in terms of $f_{q}$ and $\vec{g}_{0}[17,18]$. The classical transport equations for sigma and quark densities $f_{\sigma}$ and $f_{q}$ are reduced to the familiar
Boltzmann equations in the rest frame of the heat bath where the four-velocity is $u_{\mu}=\{1, \overrightarrow{0}\}$,

$$
\partial_{t} f_{\sigma, q}+\left(\frac{\mathbf{p} \cdot \nabla}{E_{\sigma, q}}\right) f_{\sigma, q}-\frac{\nabla m_{\sigma, q}^{2}}{2 E_{\sigma, q}} \cdot \nabla_{p} f_{\sigma, q}=-\frac{f_{\sigma, q}-f_{\sigma, q}^{t h}}{\theta}
$$

with the particle energies $E_{\sigma, q}=\sqrt{m_{\sigma, q}^{2}+p^{2}}$. The relations between the scalar densities and number densities are

$$
\begin{aligned}
& \left\langle\hat{\sigma}^{\prime} \hat{\sigma}^{\prime}\right\rangle=\int \frac{d^{3} \mathbf{p}}{(2 \pi)^{3}} \frac{1}{E_{\sigma}} f_{\sigma}(x, \mathbf{p}), \\
& \langle\hat{\bar{\psi}} \hat{\psi}\rangle=m_{q} \int \frac{d^{3} \mathbf{p}}{(2 \pi)^{3}} \frac{1}{E_{q}} f_{q}(x, \mathbf{p}) .
\end{aligned}
$$

The equilibrium distribution functions in the classical transport equations (15) are the familiar Bose-Einstein and Fermi-Dirac distributions

$$
f_{\sigma, q}^{t h}(x, \mathbf{p})=\frac{g_{\sigma, q}}{e^{\frac{E_{\sigma, q}}{T}} \mp 1}
$$

with the sigma and quark degenerates $g_{\sigma}=1$ and $g_{q}=$ 24.

The classical transport equations can be greatly simplified by taking into account Bjorken's boost invariant picture often used to describe longitudinal expansion of relativistic heavy ion collisions where a central plateau of the final rapidity distribution exists [22]. Baym extended Bjorken's method of scalar hydrodynamics to the phase space and solved the Vlasov-Boltzmann equation with a constant particle mass [24].

Neglecting transverse expansion of the system and assuming boost invariance along the longitudinal direction make the transport equations (15) for sigma and quark with effective masses much more simple, depending on the proper time $\tau=\sqrt{t^{2}-z^{2}}$ only,

$$
\partial_{\tau} f_{\sigma, q}=-\frac{f_{\sigma, q}-f_{\sigma, q}^{t h}}{\theta}
$$

with the solutions

$$
\begin{aligned}
f_{\sigma, q}(\tau, \mathbf{p}) & =e^{-\int_{\tau_{0}}^{\tau} \frac{d \tau^{\prime}}{\theta\left(\tau^{\prime}\right)}} f_{\sigma, q}\left(\tau_{0}, p_{T}, p_{z} \frac{\tau}{\tau_{0}}\right) \\
& +\int_{\tau_{0}}^{\tau} \frac{d \tau^{\prime}}{\theta\left(\tau^{\prime}\right)} e^{-\int_{\tau^{\prime}}^{\tau} \frac{d \tau^{\prime \prime}}{\theta\left(\tau^{\prime \prime}\right)}} f_{\sigma, q}^{t h}\left(T, p_{T}, p_{z} \frac{\tau}{\tau^{\prime}}\right),
\end{aligned}
$$

where the time ratios $\frac{\tau}{\tau_{0}}$ and $\frac{\tau}{\tau^{\prime}}$ come from the longitudinal expansion of the system [24].

While the quark distribution in (19) is the same as that obtained in Baym scenario [24], the quark mass here depends on the mean field $\sigma$. This dependence couples the quark distribution $f_{q}$ with the meson distribution $f_{\sigma}$ and the mean field $\sigma$. It is this coupling that leads to the phase transition from confinement to deconfinement through the change of the quark mass with the 
mean field. In the longitudinal boost invariant picture, the equation (9) of motion for the scalar condensate $\sigma$ is simplified as a normal second-order derivative equation,

$$
\left(\partial_{\tau}^{2}+\frac{1}{\tau} \partial_{\tau}\right) \sigma+\frac{\partial U}{\partial \sigma}+\frac{1}{2}\left\langle\hat{\sigma}^{\prime} \hat{\sigma}^{\prime}\right\rangle(b+c \sigma)+g\langle\hat{\bar{\psi}} \hat{\psi}\rangle=0
$$

The proper time dependence of the temperature, $T(\tau)$, in the equilibrium distribution $f^{t h}$ is determined by the energy conservation law in collisions,

$$
\begin{aligned}
& \epsilon(\tau, \sigma)=\epsilon^{t h}(T, \sigma), \\
& \epsilon(\tau, \sigma)=\int \frac{d^{3} \mathbf{p}}{(\mathbf{2} \pi)^{3}}\left[E_{\sigma} f_{\sigma}(\tau, \sigma, \vec{p})+E_{q} f_{q}(\tau, \sigma, \vec{p})\right], \\
& \epsilon^{t h}(T, \sigma)=\int \frac{d^{3} \mathbf{p}}{(\mathbf{2} \pi)^{3}}\left[E_{\sigma} f_{\sigma}^{t h}(T, \sigma, \vec{p})+E_{q} f_{q}^{t h}(T, \sigma, \vec{p})\right],
\end{aligned}
$$

where we kept only the particle contribution to the energy density, and neglected the mean field terms in $\epsilon$ and $\epsilon^{t h}$, since they are the same and do not affect the energy conservation law.

In the relaxation time approximation, the key step is the computation of the relaxation time $\theta$. In principle, $\theta$ depends on the type of particles, sigma or quark, and is a function of phase space coordinates. For all the discussions above we have neglected its type dependence and momentum dependence. Qualitatively, $\theta$ has the order of the standard strong interaction scale, $\theta \sim 1 \mathrm{fm}[24]$. Considering the fact that collision terms are the driving force for the system to reach equilibrium, they affect the system strongly only in the beginning of the evolution and are damped with increasing time. With this feature in mind we model the relaxation time by a simple step function,

$$
\frac{1}{\theta(\tau)}=\frac{1}{\lambda} \Theta\left(\tau_{e}-\tau\right)
$$

where $\lambda$ is an inverse measure of the collision strength and $\tau_{e}$ indicates the collision duration scale. $\lambda$ has the order of $1 \mathrm{fm}$ and $\lambda \rightarrow \infty$ means equilibrium without collision. In order to get a more realistic parametrization we replace in the following numerical calculations the $\Theta$ function by a more gradual exponential function $\left(1+e^{\frac{\tau-\tau_{e}}{l}}\right)^{-1}$ with the collision duration variance $l$. When $l \rightarrow 0$, it goes back to the step function.

In order to solve numerically the transport equations for the distributions $f_{\sigma, q}$ and the Klein-Gordon equation for the mean field $\sigma$ as functions of proper time $\tau$, one must know their initial values. Since the particles produced in relativistic heavy ion collisions at initial time $\tau_{0}$ are essentially emitted from the colliding point $z=0$ at $t=0$, the initial distributions in the central slice are peaked in the plane $p_{z}=0$ [24]. Therefore, we can choose the initial distributions as

$$
f_{\sigma, q}\left(\tau_{0}, \vec{p}\right)=f_{\sigma, q}^{t h}\left(T_{0}, \vec{p}\right) \delta\left(p_{z}\right)
$$

With the known number distributions (19) one can get the scalar condensates $\left\langle\hat{\sigma}^{\prime} \hat{\sigma}^{\prime}\right\rangle$ and $\langle\hat{\bar{\psi}} \hat{\psi}\rangle$ through the relations (16) and in turn the effective quark potential $U_{\text {eff }}$.

As we discussed above, there are two minima of the potential in the vacuum without collective motion, $\sigma_{p h y}$ is the global minimum but $\sigma_{p e r}$ is only the local minimum, see the dashed line in Fig.1. This means that the system is in physical vacuum. Since we focus in this paper on the phase transition from deconfinement to confinement, which can be realized in relativistic heavy ion collisions, we put initially the system in a deconfinement state with strong enough collective motion, and then study when the phase transition happens. To this end, the temperature $T_{0}$ in the initial distributions (23) should be high enough to guarantee that the system is in perturbative vacuum initially, namely $\sigma_{p e r}$ is the absolute minimum of the effective potential in the beginning. Due to the expansion and the collision terms, the energy density and effective temperature of the system fall down, and the potential difference between the physical and perturbative vacua decreases during the evolution. At some critical time $\tau_{c}$ the difference between the two vacua disappears and a first-order phase transition begins.

We define $\tau_{h}$ as the terminating time of the first-order phase transition. During the process of the phase transition, the deconfinement state and confinement state coexist, the energy densities can be expressed as

$$
\begin{aligned}
\epsilon(\tau, \sigma) & =x(\tau) \epsilon\left(\tau, \sigma_{p e r}\right)+(1-x(\tau)) \epsilon\left(\tau, \sigma_{p h y}\right), \\
\epsilon^{t h}\left(T_{c}, \sigma\right) & =x(\tau) \epsilon^{t h}\left(T_{c}, \sigma_{p e r}\right)+(1-x(\tau)) \epsilon^{t h}\left(T_{c}, \sigma_{p h y}\right),
\end{aligned}
$$

where $x(\tau)$ is the fraction of matter in the deconfinement phase at time $\tau_{c}<\tau<\tau_{h}$. From the energy conservation,

$$
\epsilon(\tau, \sigma)=\epsilon^{t h}\left(T_{c}, \sigma\right)
$$

one reads

$$
\begin{aligned}
& x(\tau)= \\
& \frac{\epsilon^{t h}\left(T_{c}, \sigma_{p h y}\right)-\epsilon\left(\tau, \sigma_{p h y}\right)}{\left[\epsilon\left(\tau, \sigma_{p e r}\right)-\epsilon^{t h}\left(T_{c}, \sigma_{p e r}\right)\right]+\left[\epsilon^{t h}\left(T_{c}, \sigma_{p h y}\right)-\epsilon\left(\tau, \sigma_{p h y}\right)\right]} .
\end{aligned}
$$

Clearly, at the beginning time $\tau_{c}$ and the end time $\tau_{h}$ of the first-order transition, one has

$$
\begin{aligned}
& x\left(\tau_{c}\right)=1, \\
& x\left(\tau_{h}\right)=0 .
\end{aligned}
$$




\section{NUMERICAL RESULTS AND DISCUSSIONS}

We choose the potential parameters [3,4,7] $a=$ $51.6 \mathrm{fm}^{-2}, b=-799.9 \mathrm{fm}^{-1}, c=4000$ and the coupling constant $g=14.8$, which are satisfactory for fitting a part of the static properties of hadrons and lead to a small bag constant $B=28 \mathrm{MeV} / \mathrm{fm}^{3}$. The temperature in the initial distributions (23) is taken as $T_{0}=160 \mathrm{MeV}$ which is high enough to guarantee an initial deconfinement state. As explained above, the collision parameter $\lambda$ describes the intensity of collisions and therefore determines how long it needs to approach the equilibrium state, and $\tau_{e}$ is the duration of the collisions which controls how long the interaction lasts. As discussed by Baym [24], $\lambda \leq 1 \mathrm{fm}$ is reasonable for strong interaction. According to the space-time scale of relativistic heavy-ion collisions, one can estimate $\tau_{e} \sim r_{0} A^{1 / 3} \mathrm{fm}$.

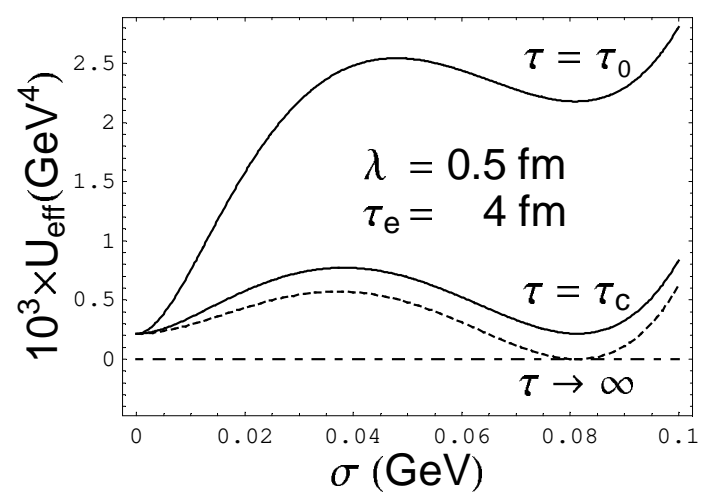

FIG. 1. The effective potential $U_{\text {eff }}$ as a function of $\sigma$ at initial time $\tau_{0}$, critical time $\tau_{c}$, and the limit $\tau \rightarrow \infty$. The deconfinement phase transition begins at $\tau_{c}=5.6 \mathrm{fm}$ and ends at $\tau_{h}=30 \mathrm{fm}$.

Fig.1 shows the effective potential $U_{\text {eff }}$ as a function of $\sigma$ at different time for collision parameters $\lambda=0.5 \mathrm{fm}$ and $\tau_{e}=4 \mathrm{fm}$. At initial time $\tau_{0}, U_{\text {eff }}$ has two minima, the local one at $\sigma=\sigma_{p h y}$ and the global one at $\sigma=\sigma_{p e r}=0$. The system stays initially in the perturbative vacuum. As time goes on, the system expands and its energy density drops down gradually. While the positions of the two minima remain unchanged during the evolution, the potential difference between the two minima, the effective bag constant $B_{\text {eff }}(\tau)$, becomes smaller and smaller. At a critical time $\tau_{c}, B_{\text {eff }}\left(\tau_{c}\right)=0$, the first-order phase transition begins and the confinement phase appears. After $\tau_{c}$, the temperature parameter in $f_{\sigma, q}^{t h}(T, \vec{p})$ remains a constant $T_{c}$, the physical vacuum and the perturbative vacuum coexist, namely the deconfinement phase and confinement phase coexist until another critical time $\tau_{h}$ when the transition is totally completed and the system is purely in confinement state. For the collision parameters used, the numerical calculation gives $\tau_{c}=5.6 \mathrm{fm}, \tau_{h}=30.4 \mathrm{fm}$, and $T_{c}=94 \mathrm{MeV}$. After $\tau_{h}$, the temperature parameter drops down again, and the global minimum is located at $\sigma=\sigma_{p h y}$. When $\tau \rightarrow \infty$, the effective potential $U_{\text {eff }}$ approaches to $U$ in the vacuum, shown as the dashed line in Fig.1. Fig.2 shows the evolution of the temperature parameter before $\tau_{c}$.

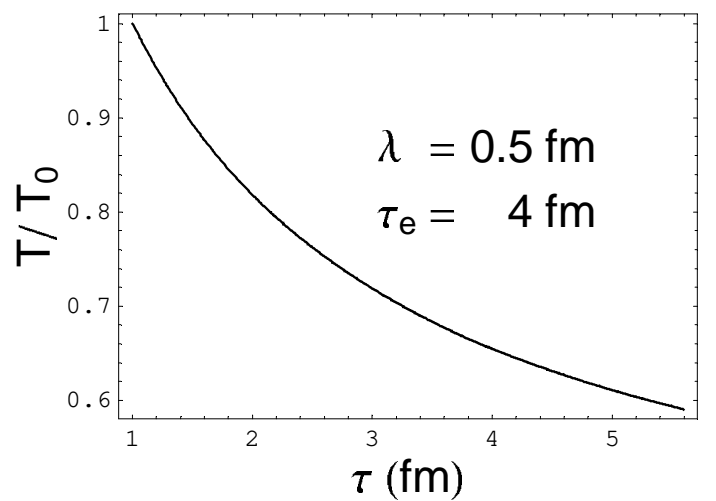

FIG. 2. The temperature parameter as a function of proper time before the phase transition.

The two critical times, $\tau_{c}$ and $\tau_{h}$, strongly depend on the relaxation time parameters, $\lambda$ and $\tau_{e}$. A system with strong interaction, namely small $\lambda$, needs only a short time to approach equilibrium state, and the temperature parameter drops down rapidly. Correspondingly, the phase transition occurs early and the duration of the first-order transition is short. The parameter $\tau_{e}$ controls also the values of $\tau_{c}$ and $\tau_{h}$. A large $\tau_{e}$ means a long last of collisions, the system needs only a short time to get to the points where the phase transition begins and ends. If the duration of the collision $\tau_{e}$ is long enough, the system will approach equilibrium state eventually. If $\tau_{e}$ is too small, however, the system will not approach equilibrium state unless the collision strength is strong enough. Fig. 3 shows the effective potential as a function of $\sigma$ in the limit of $\lambda \rightarrow \infty$. In this case, the only driving force of the phase transition is the cooling of the mean field system, the dynamics is purely reflected in the effective particle masses $m_{\sigma}$ and $m_{q}$. The transport equations (19) are reduced to

$$
f_{\sigma, q}(\tau, \mathbf{p})=f_{\sigma, q}\left(\tau_{0}, \vec{p}_{T}, p_{z} \frac{\tau}{\tau_{0}}\right) .
$$

If we neglect the collective motion of $\sigma$ since it is heavy enough, and consider the fact that before the phase transition quarks are massless in the deconfinement state, the energy conservation law in the limit of $\lambda \rightarrow \infty$ gives approximately the time evolution of the temperature parameter, $T(\tau) \sim T_{0}\left(\tau_{0} / \tau\right)^{1 / 4}$. Only when the thermal distribution in (23) is taken as Boltzmann distribution, we have exactly,

$$
T(\tau)=T_{0}\left(\frac{\tau_{0}}{\tau}\right)^{1 / 4} .
$$

If we put the initial state in a 3 -dimensional equilibrium state and take the mass to be zero, the longitudinal 
expansion of the system will force the state in nonequilibrium afterwards. The energy conservation in the limit of no collisions leads to

$$
\begin{aligned}
& T(\tau)=T_{0}\left(\frac{\tau_{0}}{\tau}\right)^{1 / 4} g(\tau) \\
& g(\tau)=\left[\frac{1}{2}\left(\frac{\tau_{0}}{\tau}+\frac{\sin ^{-1} \sqrt{1-\left(\frac{\tau_{0}}{\tau}\right)^{2}}}{\sqrt{1-\left(\frac{\tau_{0}}{\tau}\right)^{2}}}\right)\right]^{\frac{1}{4}} .
\end{aligned}
$$

Since the quark mass is taken as a constant, $m=0$ in this special case, the quark distribution is decoupled from the meson distribution and the mean field, the system is reduced to the case in Baym scenario. Therefore, the result (30) has a similar structure to eq. (19) of the work by Baym [24].

The comparison between (29) and (30) is shown in Fig.4. Since the function $g(\tau) \leq 1$, the temperature with initial equilibrium state is always lower than that with initial nonequilibrium state for any time $\tau>\tau_{0}$.

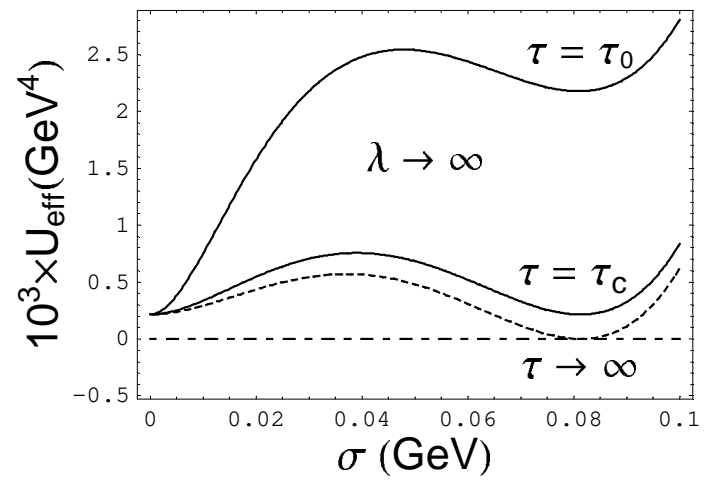

FIG. 3. The effective potential $U_{\text {eff }}$ as a function of $\sigma$ at initial time $\tau_{0}$, critical time $\tau_{c}$, and the limit $\tau \rightarrow \infty$ in the case without collisions. In this limit, the phase transition starts at $\tau_{c}=10 \mathrm{fm}$ and ends at $\tau_{h}=841 \mathrm{fm}$.

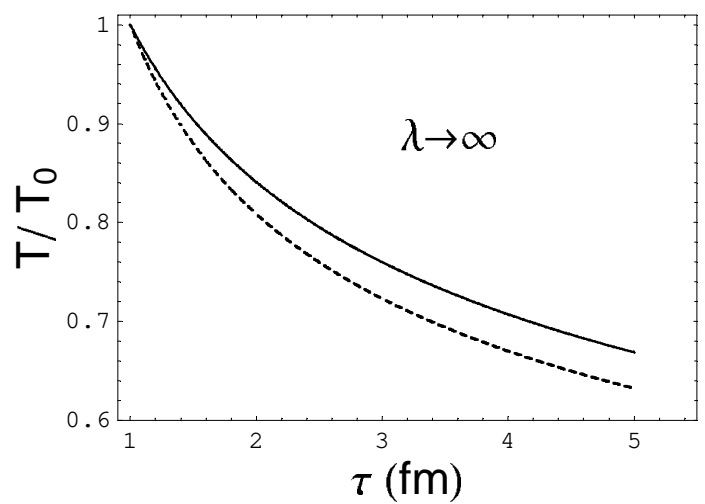

FIG. 4. The time evolution of the temperature parameter with initial nonequilibrium state (solid line) and with initial equilibrium state (dashed line) in the limit of no collisions.

While the collisions do not change the critical value of the temperature parameter remarkably, it is $94 \mathrm{MeV}$ for $\lambda=0.5 \mathrm{fm}$ and $\tau_{e}=4 \mathrm{fm}$ and $89 \mathrm{MeV}$ for $\lambda \rightarrow \infty$, the time structure of the phase transition is controlled by the collisions. The relaxation time dependence of the critical time $\tau_{c}$ and the duration $\Delta \tau=\tau_{h}-\tau_{c}$ scaled by their values in the limit of no collisions is shown in Figs. 5 and 6. Both decrease monotonously with increasing collision strength and/or increasing collision duration. When the collisions are strong enough and the collision duration is long enough, the phase transition will start before the collisions cease. This is clearly reflected in Fig.5 where $\tau_{c}$ at $\lambda=0.2 \mathrm{fm}$ no more changes when $\tau_{e}>4 \mathrm{fm}$. With increasing $\lambda$ the starting time $\tau_{c}$ and the duration $\Delta \tau$ will approach finally the limit values of no collisions, they converge at $10.12 \mathrm{fm}$ and $831 \mathrm{fm}$, respectively. The speed of the convergence is governed by the collision duration. When the collision duration is short enough, the system is not sensitive to the collisions in the region of weak strength. This is the reason why $\tau_{c}$ and $\Delta \tau$ converge very fast with small $\tau_{e}$.

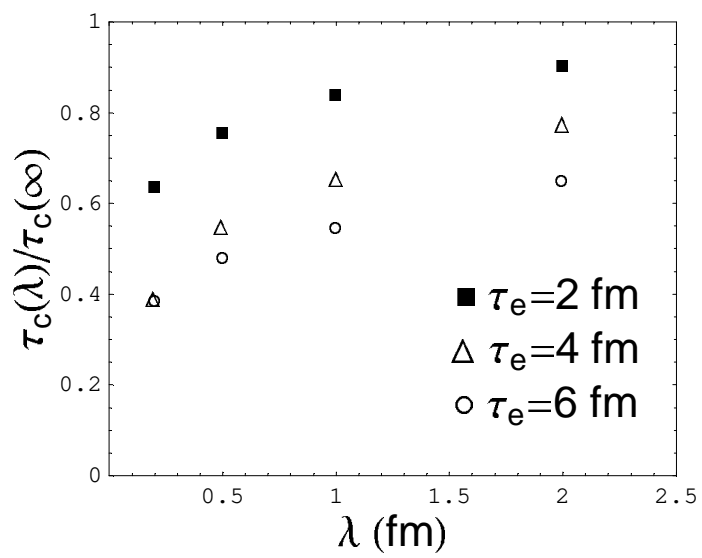

FIG. 5. The beginning time of first-order phase transition scaled by its limit value without collisions as a function of the collision strength for collision duration $\tau_{e}=2,4,6 \mathrm{fm}$.

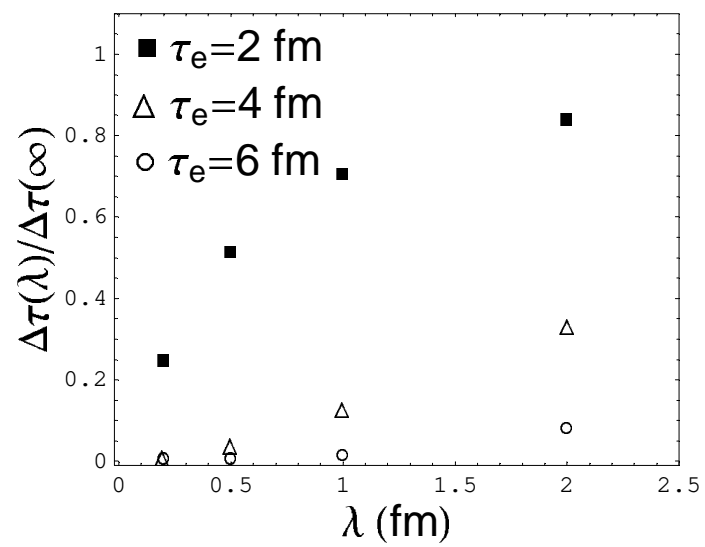

FIG. 6. The duration of the first-order phase transition scaled by its limit value without collisions as a function of the strength for collision duration $\tau_{e}=2,4,6 \mathrm{fm}$.

Fig.7 shows the temperature parameter as a function 
of time for fixed collision duration $\tau_{e}=4$ fm but different values of collision strength. Each line starts at initial time $\tau_{0}=1 \mathrm{fm}$ and ends at the critical time $\tau_{c}$ which depends on the collision strength. After $\tau_{c}$ the temperature does not change until the second critical time $\tau_{h}$. With increasing collision strength, namely decreasing $\lambda$, the system cools down more and more fast, and the phase transition happens more and more early.

In principle, only one of the two collision parameters $\lambda$ and $\tau_{e}$ is free. A system with strong interaction needs a short time to approach equilibrium, and vice versa. While it is difficult to determine the relation between the two parameters in relaxation time approximation, we can obtain a constraint condition. For a system with fixed collision duration scale $\tau_{e}$, the collision strength should larger than the maximum value corresponding to the well-known Bjorken scaling solution $T / T_{0}=\left(\tau_{0} / \tau\right)^{1 / 3}[22]$. Since $\lambda$ is the inverse measure of the collision strength, we have the constraint

$$
\lambda_{\min }<\lambda \text {. }
$$

For $\tau_{e}=4 \mathrm{fm}, \lambda_{\min }$ is approximately $0.3 \mathrm{fm}$. When $\lambda$ is less than $\lambda_{\min }$, the result will not be physical.

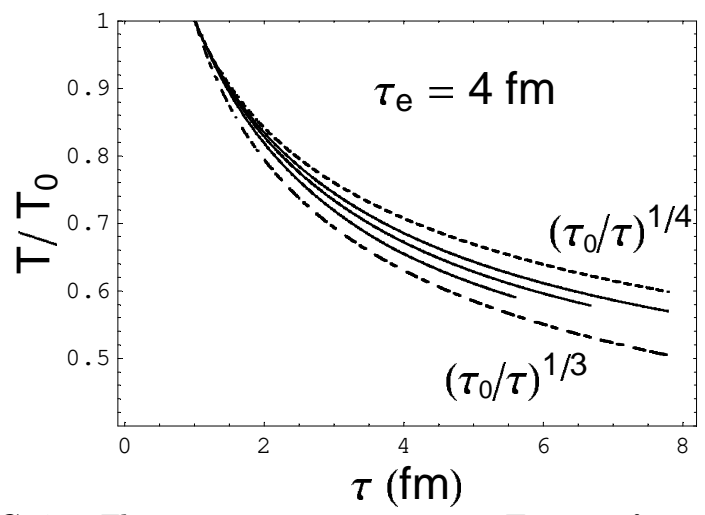

FIG. 7. The temperature parameter $T$ as a function of proper time $\tau$ at fixed $\tau_{e}$. The solid curves from the bottom to the top correspond to $\lambda=0.5,1$ and $2 \mathrm{fm}$, and the two dashed lines correspond to the limit (29) and the Bjorken limit, respectively.

\section{SUMMARY}

We investigated the deconfinement phase transition of an expanding quark system in Friedberg-Lee model, considering the collision terms in relaxation time approximation. We calculated the beginning time and the duration of the first-order phase transition for different collision strength. While the critical temperature of the phase transition is dominated by mean field, described by $\lambda \rightarrow \infty$ in our treatment, the collision terms have significant influence on the beginning time and the duration of the transition. Strong collisions result in an early and short transition, and weak collisions make the transition begin late and last a long time. Although our results are derived in a particular model, we expect the qualitative dependence of the phase transition times on the collisions to be of more general validity, because it is well known that collisions are the driving force of thermalization and control the speed of thermalization process of any system.

Since the deconfinement phase is only an intermediate state in relativistic heavy ion collisions, there is no way to measure its properties directly. One needs to extract signatures of the phase transition and the new state from analysis of final state distributions of different particles, such as low moment dilepton enhancement, $J / \Psi$ suppression and strangeness enhancement at SPS [25] and disappearance of back-to-back jets at RHIC [26]. Normally the theoretical study on these signatures is based on the assumption of equilibrium system. According to our discussion above, the thermalization process due to collisions among the particles make the phase transition happen early and shorten the duration of the first-order phase transition. As a consequence, the contribution from the deconfinement phase and the coexisting phase to the final state distributions will be changed, especially for those signatures extracted from the early evolution.

Acknowledgments: We thank Larry Mclerran who drew our attention to the time dependence of collision terms. We are grateful to Jisheng Chen, Jinfeng Liao and Xianglei Zhu for many discussions during the work. The work was supported in part by the grants NSFC19925519, 10135030, and G2000077407.

[1] R. Friedberg and T. D. Lee, Phys. Rev. D15, 1694(1977); 16, 1096(1977); 18, 2623(1978).

[2] T. D. Lee and Y, Pang, Phys. Rept. 221, 251(1992).

[3] L. Wilets, Nontopological Solitions, World Scientific, Singapore(1989).

[4] R. Goldflam and L. Wilets, Phys. Rev. D25, 1951(1982).

[5] H. Reinhardt, B. V. Dang and H. Schulz, Phys. Lett. 159B, 161(1985).

[6] Ming Li, M. C. Birse and L. Wilets, J. Phys. G 13, 1(1987).

[7] Wang Enke, Li Jiarong and Liu Lianshou, Phys. Rev. D41, 2288(1990).

[8] Gao Song, Wang Enke and Li Jiarong, Phys. Rev. D46, 3211(1992).

[9] For instance, see, F. Karsch, Nucl. Phys. A698, 199(2002).

[10] For a review, see, H.-Th. Elze and U. Heinz, Phys. Rep. 183, 81(1989).

[11] U. Heinz, Phys. Rev. Lett. 51, 351(1983).

[12] U. Heinz, Ann. Phys. (N.Y.) 161, 48(1985).

[13] H.-Th. Elze, M. Gyulassy, and D. Vasak, Nucl. Phys. B276, 706(1986). 
[14] H.-Th. Elze, M. Gyulassy, and D. Vasak, Phys. Lett. B177, 402(1986).

[15] S. R. de Groot, W. A. van Leeuwen, and Ch. G. van Weert, "Relativistic Kinetic Theory", North-Holland, Amsterdam, 1980.

[16] I. Bialynicki-Birula, P. Gornicki, and J. Rafelski, Phys. Rev. D44, 1825(1991).

[17] P. Zhuang, and U. Heinz, Ann. Phys. (N.Y.) 245, 311(1996).

[18] P. Zhuang, and U. Heinz, Phys. Rev. D57, 6525(1998).

[19] S. Ochs, and U. Heinz, Ann. Phys. (N.Y.) 266, 351(1998).

[20] U. Kalmbach, T. Vetter, T.S. Biro and U. Mosel, Nucl. Phys. A563, 584(1993).

[21] T. Vetter, T.S. Biro and U. Mosel, Nucl. Phys. A581, 598(1995).

[22] J. D. Bjorken, Phys. Rev. D27, 140(1983).

[23] Q. Haider, L.C. Liu J.Phys. G21, 589(1995).

[24] G. Baym, Phys. Lett. B138, 18(1984).

[25] For instance, see, U. Heinz, Nucl. Phys. A685, 414(2001).

[26] For instance, see, J. Adams, et al. (STAR Collaboration), Phys. Rev. Lett. 91, 072304(2003). 